\title{
Size effects on switching field of ring-shaped permalloy elements
}

\author{
C.C. Chang ${ }^{\mathrm{a}, \mathrm{b}}$, Y.C. Chang ${ }^{\mathrm{a}, \mathrm{b}}$, J.C. Wu ${ }^{\mathrm{a}, \mathrm{b}, *}$, Z.H. Wei ${ }^{\mathrm{c}}$, M.F. Lai ${ }^{\mathrm{c}}$, C.R. Chang, \\ J.H. Kuo ${ }^{\mathrm{d}}$ \\ ${ }^{a}$ Taiwan SPIN Research Center, National Changhua University of Education, Changhua 500, Taiwan \\ ${ }^{\mathrm{b}}$ Department of Physics, National Changhua University of Education, Changhua 500, Taiwan \\ ${ }^{\mathrm{c}}$ Department of Physics, National Taiwan University, Taipei 10617, Taiwan \\ ${ }^{\mathrm{d}}$ Institue of Physics, Academia Sinica, Taipei 115, Taiwan
}

Available online 10 May 2004

\begin{abstract}
The magnetization switching behavior of permalloy ring elements as a function of ring diameter and film thickness by magnetoresistance measurement is presented. The permalloy ring elements, with diameters of $2-5 \mu \mathrm{m}$ and a thickness of 14-66 nm, were fabricated by electron beam lithography through a lift-off process. The switching field for the transition from the vortex state to the onion state increases with decreasing size. Furthermore, the switching field increases with increasing thickness up to a critical value of about $53-65 \mathrm{~nm}$, over which the switching field tends to decrease with increasing thickness.
\end{abstract}

(C) 2004 Elsevier B.V. All rights reserved.

PACS: 75.60.-d; 70.50.Cc

Keywords: Permalloy ring; Magnetoresistance; Coherent rotation

Generally, the geometry determines which kind of magnetization configuration and switching mechanism may be present. Many investigations have been carried out on elements with simple geometries, such as squares [1], disks [2], rectangles [3], and rings [4]. Among various shapes of magnetic elements, the ring-shaped element has become a promising candidate for the magnetic

\footnotetext{
*Corresponding author. Taiwan Spin Research Center, National Changhua University of Education, Changhua 500, Taiwan. Tel.: +886-4-7232105x3343; fax: +886-4-7211153.

E-mail address: phjcwu@cc.ncue.edu.tw (J.C. Wu).
}

random access memory [5] or sensor devices [6]. The application of such elements requires the simplest, fastest, and most reproducible switching mechanism. The advantages of ring-shaped element are less field leakage and influence of edge domains. The data storage density of the devices and the uniformity of switching process can be thus enhanced. In order to investigate the magnetization switching mechanism, some approaches, such as magneto-optic Kerr effect (MOKE) [7], vibrating sample magnetometers (VSM), and scanning Hall probe microscope (SHPM) [8] are generally used. Moreover, for ring-shaped 
elements, the magnetoresistance behavior corresponding to different magnetization configurations had been investigated by experiments and simulations [9-12]. Herein, we present a systematic study of the switching field contribution from the vortex state to the onion state transition as a function of the ring diameter and film thickness.

Four-terminal micron-sized permalloy ring devices were fabricated by a two-step standard electron beam lithography and thermal evaporation through a lift-off technique. For investigating the size dependence of magnetoresistance, the permalloy ring elements with diameters of $2,3,4$, and $5 \mu \mathrm{m}$, and linewidth of $0.4,0.6,0.8,1 \mu \mathrm{m}$, respectively, were fabricated. Furthermore, for investigating the thickness dependence, the permalloy ring elements with $2 \mu \mathrm{m}$ diameter, and varying the thickness as $14,30,33,46,53$, and $66 \mathrm{~nm}$ were fabricated. Fig. 1 shows an atomic force microscope (AFM) image of a permalloy ring with $3 \mu \mathrm{m}$ diameter and $46 \mathrm{~nm}$ thickness patched by $\mathrm{Au}$ current leads.

Magnetoresistance measurements were carried out with $20 \mu \mathrm{A}$ dc sensing current and under an external magnetic field applied in the film plane transverse to the current between electrodes. Fig. 2 shows the magnetoresistance curve for the $2 \mu \mathrm{m}$ diameter and $30 \mathrm{~nm}$ thickness ring under an applied field from -1.2 to $1.2 \mathrm{kOe}$ (following the solid arrows). The complete reversal process takes place via three transitions. The first transition occurs near positive zero fields; the configuration changes from an onion configuration at large negative fields into a vortex-pair configuration.

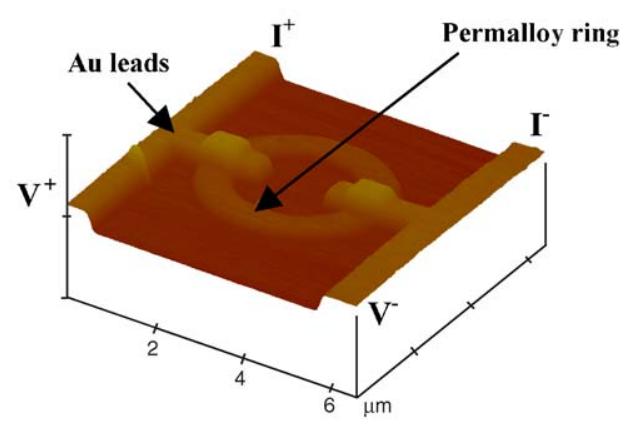

Fig. 1. Atomic force microscope image of a four-terminal permalloy ring device ( $3 \mu \mathrm{m}$ diameter and $46 \mathrm{~nm}$ thickness).

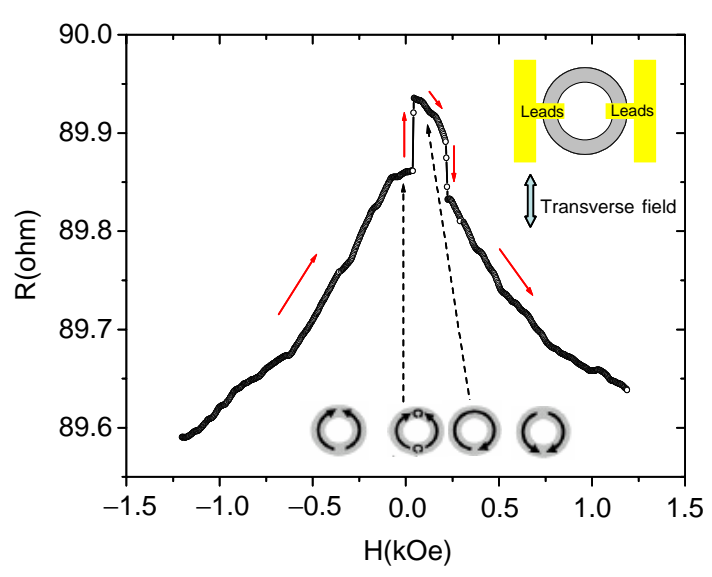

Fig. 2. Magnetoresistance curve of a $2 \mu \mathrm{m}$ diameter ring under an applied field in the film plane as shown in upper inset. The lower inset shows the switching process of magnetization configuration.

The second transition occurs at small positive fields and the configuration changes from the vortex-pair configuration into a vortex configuration. Finally, the third transition occurs at large positive fields and the configuration changes from the vortex configuration into an opposite onion configuration [9]. The higher magnetoresistance value occurs at the vortex configuration of magnetization, as shown in Fig. 2, due to the well-known anisotropic magnetic effect.

In this paper, we present the relationship between ring diameter/thickness and the switching field for the transition from the vortex state to the onion state. Fig. 3 shows the experimental magnetoresistance curves of permally rings of four different sizes but keeping the same thickness of $46 \mathrm{~nm}$ and aspect ratio of 5 , which is defined by a ratio of diameter to linewidth. Fig. 4 shows the analysis of the switching field with respect to the ring diameter, extracted from Fig. 3. Notice that, the switching field for vortex to onion transition decreases with increasing ring size, the experimental result is in good agreement with micromagnetic simulation [9]. It is well known that the vortex structure decreases the demagnetization energy, and the two-vortex-pair state occurs more easily in larger rings. So the total energy decreases with increasing ring size even though there is a small increase in the exchange energy, as shown in 


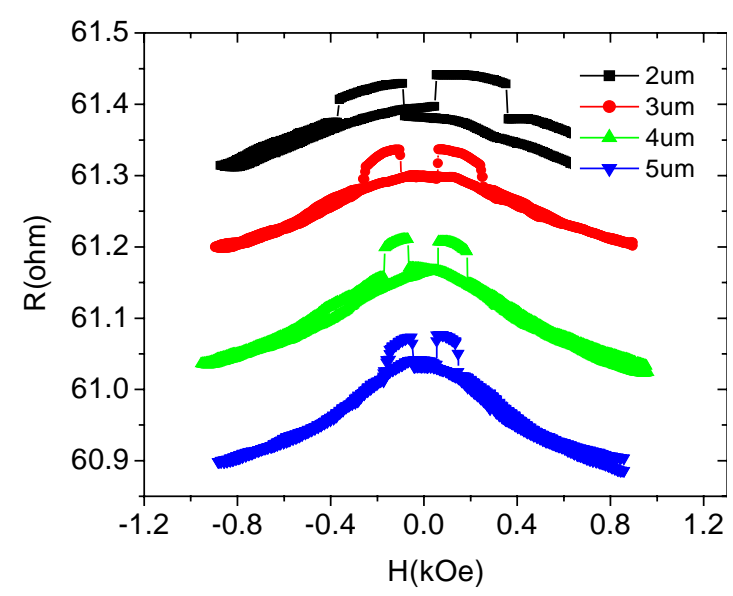

Fig. 3. Magnetoresistance curves of four different sizes (2$5 \mu \mathrm{m})$ and $46 \mathrm{~nm}$ thickness rings, the curves are offset vertically for clarity.

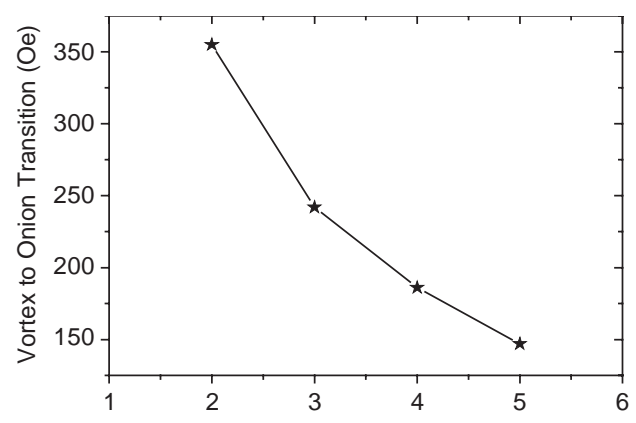

Fig. 4. The switching field for vortex to onion transition is a function of ring diameter, the data are extracted from Fig. 3.

Fig. 4, i.e. the overall coercivity is decreased in larger rings.

The thickness dependence of the switching field for vortex to onion transition is showed in Fig. 5. The switching field first increases monotonically until a critical thickness $t_{\mathrm{c}}$ between 53 and $65 \mathrm{~nm}$, over which the switching field tends to decrease with increasing thickness. These two different tendencies stand for different magnetization reversal mechanisms. Since the demagnetization energy increases with increasing thickness, the magnetization reversal is more likely to be coherent rotation in a thinner ring, i.e. the total energy increases with increasing thickness. However, a

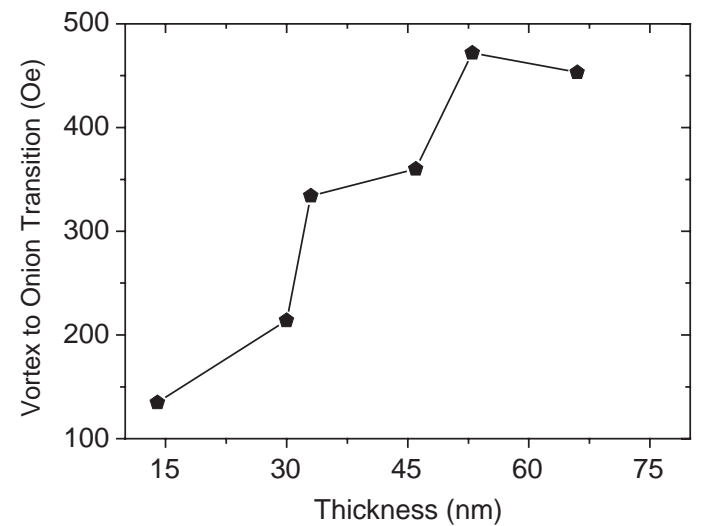

Fig. 5. The thickness dependence of the switching field for vortex to onion transition. The maximum switching field occurs at the range of thickness from 53 to $65 \mathrm{~nm}$.

vortex structure tends to exist easily in a thicker film. When the thickness is above the critical thickness, the vortex can reduce the demagnetization energy more efficiently in the thicker rings; consequently, the switching field decreases with increasing thickness as the thickness is above the critical thickness.

In conclusion, we have studied the switching field dependence of permalloy ring fabricated by electron beam lithography through a lift-off process. We find that the switching field for the transition from the vortex state to the onion state is a function of ring diameter and thickness. For size dependence of the switching field, the vortex structure decreases the demagnetization energy of the wider ring. So the switching field decreases with increasing size. For thickness dependence of the switching field, the switching field increases with increasing thickness below the critical thickness, due to coherent rotation; the switching field decreases with increasing thickness above the critical thickness, due to the reduction of demagnetization energy by the vortex structure in the thicker film. The critical thickness of the rotation mechanism is between 53 and $65 \mathrm{~nm}$ for the ring of $2 \mu \mathrm{m}$ diameter and of $0.4 \mu \mathrm{m}$ width.

This work was supported by the Ministry of Economic Affairs with grant no. 92-EC-17-A-01S1-026, and partially by National Science Council 
grant with no. 92-2120-M-018-001. In addition, we would like to thank Jun-Yang Lai for useful discussions.

\section{References}

[1] R.P. Cowburn, A.O. Adeyeye, M.E. Welland, Phys. Rev. Lett. 81 (1998) 5414.

[2] R.P. Cowburn, D.K. Koltsov, A.O. Adeyeye, M.E. Welland, D.M. Tricker, Phys. Rev. Lett. 83 (1999) 1042.

[3] Y. Zheng, J.G. Zhu, J. Appl. Phys. 81 (1997) 5471.

[4] S.P. Li, D. Peyrade, M. Natali, A. Lebib, Y. Chen, U. Ebels, L.d. Buda, K. Ounadjela, Phys. Rev. Lett. 86 (2001) 1102.
[5] J.G. Zhu, Y. Zheng, G.A. Prinz, J. Appl. Phys. 87 (2000) 6668.

[6] M.M. Miller, G.A. Prinz, S.F. Cheng, S. Bounnak, Appl. Phys. Lett. 81 (2002) 2211.

[7] M. Kläui, J. Rothman, L. Lopez Diaz, C.A.F. Vaz, J.A.C. Bland, Z. Cui, Appl. Phys. Lett. 78 (2001) 3268.

[8] J. Bekaert, D. Buntinx, C. Van Haesendonck, V.V. Moshchalkov, J. De Boeck, G. Borghs, V. Metlushko, Appl. Phys. Lett. 81 (2002) 3413.

[9] M. Kläui, C.A.F. Vaz, J.A.C. Bland, W. Wernsdorfer, G. Faini, E. Cambril, Appl. Phys. Lett. 81 (2002) 108.

[10] M.F. Lai, Z.H. Wei, C.R. Chang, J.C. Wu, J.H. Kuo, J.Y. Lai, Phys. Rev. B 67 (2003) 104419.

[11] M. Kläui, C.A.F. Vaz, J.A.C. Bland, W. Wernsdorfer, G. Faini, E. Cambril, Phys. Rev. Lett 90 (2003) 097202.

[12] M. Kläui, C.A.F. Vaz, J.A.C. Bland, W. Wernsdorfer, G. Faini, E. Cambril, J. Appl. Phys. 93 (2003) 7885. 